\title{
Face Recognition Technology Based On Image Processing
}

\author{
Chen Xin, Yajuan Li, Zhimin Tian \\ Hebei Engineering and Technical College, Cangzhou, China \\ 54892713@163.com
}

Keywords: Face recognition, Image processing, Face detection, Facial features

\begin{abstract}
Face recognition is an important identification technology, which has broad application prospects. As an important biometric feature with high universal and non-contact collecting, face is increasingly being used for authentication. This paper describes the basic process of face recognition and briefly analyzes the image processing technologies in face recognition, such as light compensation, Gaussian smoothing, similarity calculation and edge detection method. Face recognition technology is also described in detail, and the eye is the most critical part in human facial features. Finally, two candidates are selected for simulation and analysis to verify the effectiveness and feasibility of image processing method.
\end{abstract}

\section{Introduction}

Privacy information security is eager to be effectively protected in contemporary society, so people need a more secure and more convenient protection measure. Traditional measures such as the presence of the key and password exist great vulnerability, and more and more people realize that such protection method is not very safe. Over the recent years, the face recognition technology has continued to make progress, which is being used from the laboratory towards real life, and plays a great role in the access control and video surveillance. Compared with other biometric technologies such as voice recognition, iris recognition, the benefits of face recognition are significant, such as difficult to counterfeit, without touching the skin, and low acquisition equipment.

Face recognition especially means the computer technology of using analysis and comparison of facial and visual features to identify the identity information. Face recognition technology is widely used, it can be used for security authentication systems, medical and file management, the monitoring system of banking and customs, and automatic access control systems. For its potential application as well as incomparable superiority, face recognition has increasingly become a hot spot in pattern recognition and artificial intelligence. But the face recognition systems based on BP neural network or PCA are expensive, bulky with high power consumption due to the complexity of the algorithms, which makes the application platform of this technology be greatly limited. Therefore, this paper is going to start from the perspective of image processing to obtain the face image from the camera and then process in order to improve the accuracy of recognition and positioning.

\section{Image Processing Methods}

The image processing methods commonly used include light compensation, Gaussian smoothing, similarity calculation and edge detection method, which can be used for face recognition. Face recognition is a promising means for man machine interface and security. For practical use of face recognition, it is necessary to overcome the variation of the face, such as facial expression, face direction, lighting condition and aging. Next, some common image processing methods are introduced in brief.

- Light compensation: Different light will affect the collected images of camera, we only need to adjust the brightness modestly, and this is the image light compensation.

- Gaussian smoothing: When you save image, the entire contents of the image cannot be saved completely, which will lead to lack of data. In order to solve this problem, we have to eliminate the 
noise by Gaussian smoothing to ensure the integrity of the image.

- Similarity calculation: Image similarity is mainly used to evaluate two images to see if they are similar in some places. For example, there are images A and B, respectively, to calculate the histogram of the two images, then calculate the normalized correlation coefficient of the two histograms such as Pap distance and histogram intersection distance. Based on the difference between the vectors calculated mathematically to estimate the image similarity degree, which is the image equalization histogram, and it can be well normalized. So the similarity of two images with different resolution can be calculated by computing the histogram, which is easy to calculate and the amount is relatively small.

- Binary: Image binary is to set pixel gray value to 0 or 255 according to threshold, namely, the entire image shows clear black and white visuals.

- Edge detection: The purpose is to identify the points with obvious brightness variation in digital image. The significant change can reflect the varied image pattern.

\section{Face Recognition Technology}

Face recognition technology is completed mainly through three steps, namely, face detection and location, facial feature extraction and face comparison and recognition precisely.Face detection is also based on a subspace method where two subspaces, the face subspace and the non-face subspace, are stored for distinguishing the face class from the non-face class. The similarities of the subspaces are calculated for each $15 \times 15$ sliding window. This calculation is done for plural scaled input images. The location of the face is determined as the position which gives the global maximum of similarity of the face class while the similarity of the face class is greater than that of the non-face class. The system uses the positions of pupils and nostrils to normalize the region for recognition. The detection method for these facial parts is based on a combination of shape extraction and pattern matching. The flow of processing can be seen in Fig. 1.

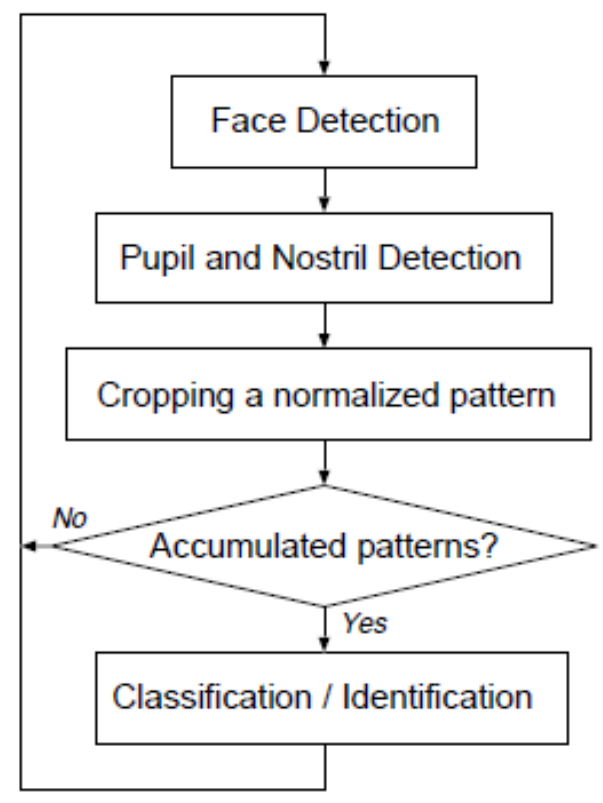

Fig.1 Flow of detection processing technology

Bio facial features can be constructed according to human organ, the most obvious features in facial features can be roughly estimated according to the valley and frequency characteristic of the gray distribution of facial features, such as eyes, nose and mouth. Through these distinct facial features, face location will be approximately determined. On this basis, initial parameters can be given to significantly improve the speed and accuracy of the algorithm. The processed image is then used for face positioning, the obvious facial features such as eyes, nose and mouth can be flagged first for comparison with other images. Finally, we can extract different eigenvalues from the image and compare them with back-end database to get the recognition results. 
The overall structure of the application design flow is shown in fig.2.

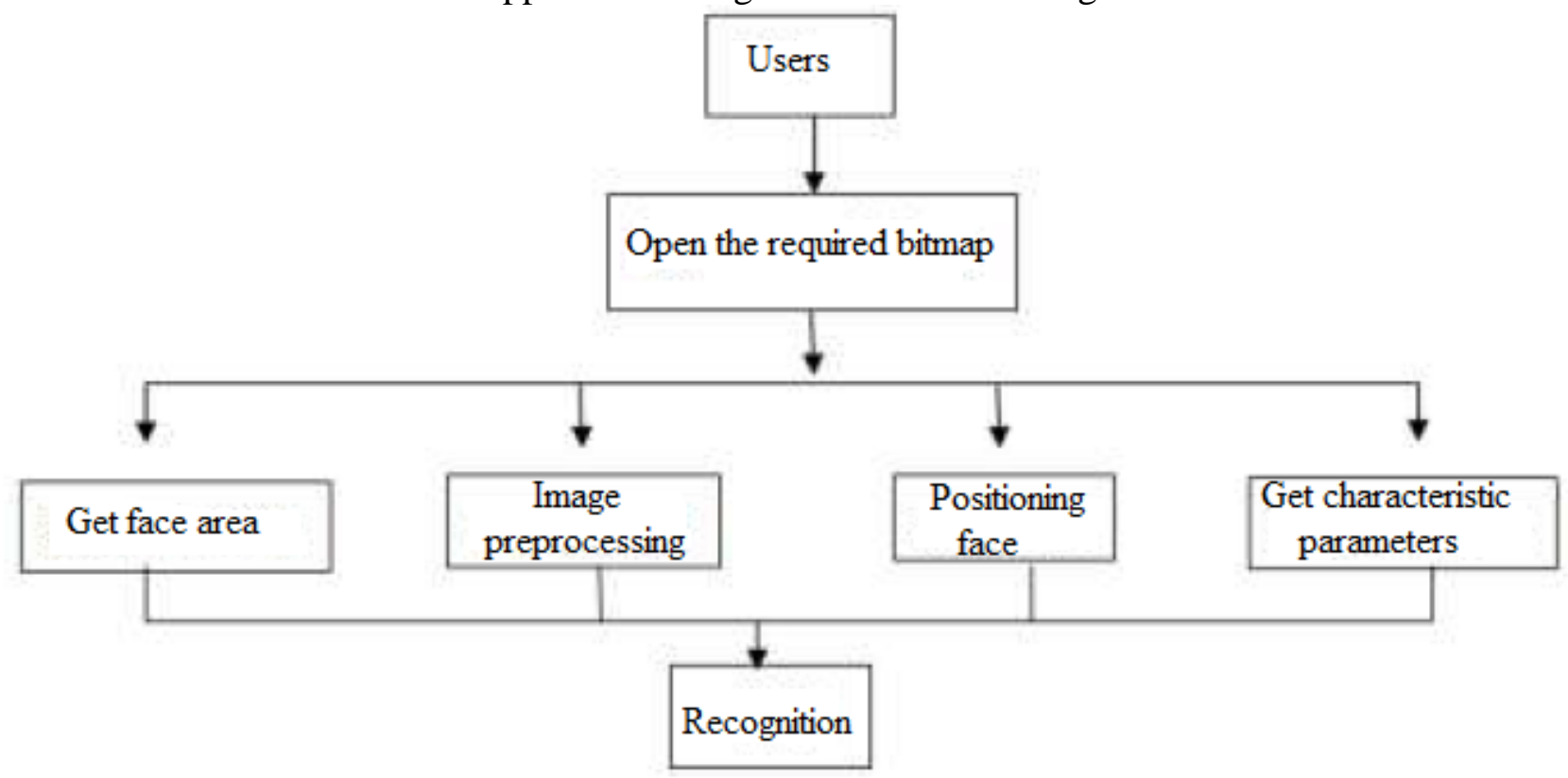

Fig.2 Overall structure of the application design flow

Face location is to mark typical facial features (eyes, nose, mouth, etc.), the eyes are the most obvious feature in facial features with its symmetry, the nose is below the middle of the two eyes, and mouth again below the nose, so we just need to mark a good eye, and nose and mouth will be better marked.

In face recognition, the eye is the most critical part in human facial features. First through the eyes positioning, it is determined whether the presence of a human face is real, so the eyes positioning is the key to face recognition. Use the existing regional growth eye positioning technology, according to the critical eye in human facial features for face positioning. Because the eyes in the human face is symmetrical, we use this feature of the eyes on the face, using different gray valley area to determine the position of the two eyes to determine the general framework of a human face, and then take advantage of regional growth of search strategy to find the position of the nose in the face by positioning the eyes out of the face frame, because the nose is just below the two eyes, it is better positioned, then define two initial search rectangle to the approximate location in which the left and right eyes growth respectively. Several tests proves that there is a strong recognition ability of image processing technology to distinguish, whether face-to-face big or small, or changes in light, but the drawback is that it will be too inaccurate when the situation of dark light leading to the emergence of gray difference between eyes and face is not very large, or the face in image is on the side very much.

\section{Simulation Analysis}

For more vivid description of the image processing in face recognition technology, we choose two candidates for analysis. Normalization is based on the extracted four feature points (pupils and nostrils). A normalized pattern image is cropped as $30 \times 30$ image by subsampling the input image. Fig.3 illustrates the normalization process. The vectors $V_{1}$ and $V_{2}$ are determined from the four points, and $E_{\mathrm{V} 1}=V_{1} / m_{1}$ and $E_{\mathrm{V} 2}=V_{2} / m_{2}$ are calculated using $m_{1}$ and $m_{2}$ as constant weights. Point $\mathrm{C}$ is the center of the two pupils. The normalized pattern is generated by subsampling the pixels of the locations. Since the motion of the mouth is an unstable feature, we determine the parameters $m_{1}$ and $m_{2}$ to discard the mouth area. 


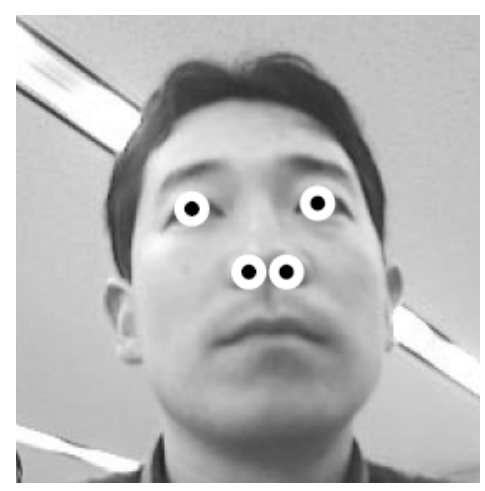

(a)

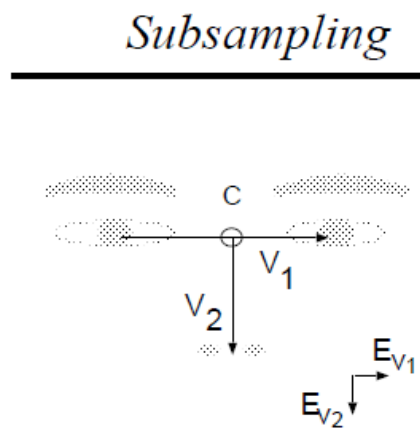

(b)

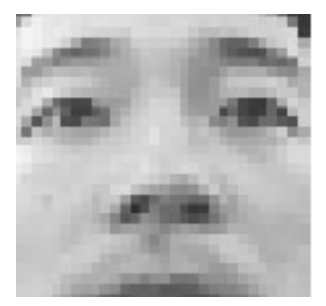

(c)

Fig.3 Crop a normalized pattern. (a) Select four feature points (b) The vectors for subsampling (c) A normalized pattern

However, the eyes location is often difficult, and there are common three cases in eyes location, namely, no eyes moved, no eyes moved and two eyes moved, which can be seen in Fig.4.

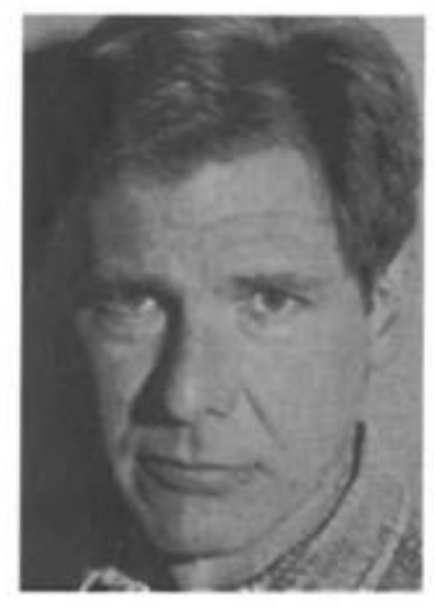

(a)

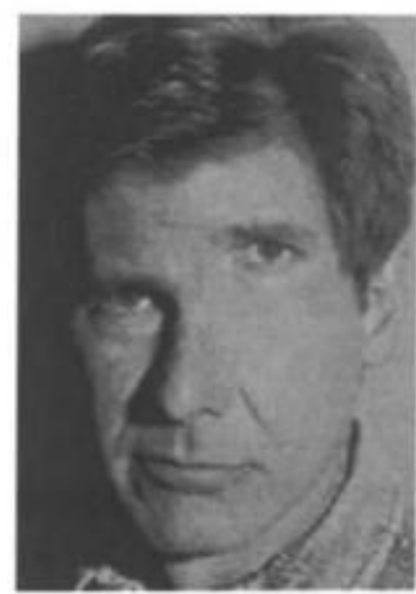

(b)

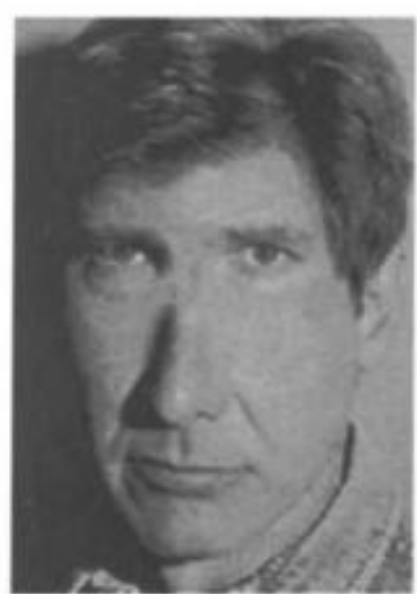

(c)

Fig.4 Three cases in eyes location. (a) No eyes moved (b) One eye moved (c) Two eyes moved

\section{Conclusions}

Face recognition technology is widely used in security authentication systems, medical and file management, the monitoring system of banking and customs, and automatic access control systems. It is completed mainly through three steps, namely, face detection and location, facial feature extraction and face comparison and recognition precisely. In this paper, we have proposed a new method for face recognition using promoted image processing method, the simulation results also verify the effectiveness and feasibility of image processing, which can provide certain references for further research.

\section{References}

[1] Yamaguchi O, Fukui $\mathrm{K}$ and Maeda $\mathrm{K}$. Face recognition using temporal image sequence]//Automatic Face and Gesture Recognition, 1998. Proceedings. Third IEEE International Conference on. IEEE, 1998: 318-323.

[2] Z.Q. Xie, J.Y. Zhou and F.Q. Lin. Face Recognition Algorithm Based on Image Processing. Computer Knowledge and Technology, 2015, 3: 090. (in Chinese)

[3] Face recognition: From theory to applications. Springer Science \& Business Media, 2012.

[4] C. Liu. Simulations for orbital maintenance of low-angle satellite based on STK. Energy Education Science and Technology Part A: Energy Science and Research, 32(6): 6307-6316. 
[5] Pavlidis T. Algorithms for graphics and image processing. Springer Science \& Business Media, 2012.

[6] B. Xiao and Y.H. Wang. Survey of Human Face Recognition. Application Research of Computers, 2005, 8:1-5. (in Chinese)

[7] Vidya V, Farheen N, Manikantan K, et al. Face recognition using threshold based DWT feature extraction and selective illumination enhancement technique. Procedia Technology, 2012, 6: 334-343.

[8] VLSI for pattern recognition and image processing. Springer Science \& Business Media, 2013.

[9] Choi J Y, Ro Y M, Plataniotis K N. Color local texture features for color face recognition. Image Processing, IEEE Transactions on, 2012, 21(3): 1366-1380. 